\title{
Atmospheric Solids Analysis Probe (ASAP) and Atmospheric Pressure Gas Chromatography (APGC) coupled to Quadrupole Time of Flight Mass Spectrometry (QTOF-MS) as alternative techniques to trace aromatic markers of mineral oils in food packaging
}

\author{
Janira Jaén, Celia Domeño, Pilar Alfaro, Cristina Nerín \\ Instituto de Investigación en Ingeniería de Aragón (I3A), Depto. Química Analítica, EINA, Universidad de Zaragoza, María de Luna 3, 50018, Zaragoza, Spain
}

\section{A R T I C L E I N F O}

\section{Keywords:}

ASAP-QTOF-MS

APGC-QTOF-M

Mineral oils

MOAH

r-PET

Markers

\begin{abstract}
A B S T R A C T
The aim of this work was to select and identify the best markers of aromatic hydrocarbon mineral oil (MOAH) in food packaging. For this purpose, a series of mineral oils was initially analysed. Polycyclic Aromatic Hydrocarbons (PAHs) and the alkylated isomers of Methylnaphthalene (MNS), Diisopropylnaphtalene (DIPNs), Dibenzothiophenes (DBTS), Methyldibenzothiophene (MDBTs), Dimethyldibenzothiophenes (DMDBTs) and Benzonaphthiophenes (BNTS) were then explored. Their presence was confirmed by direct analysis of several mineral oils by Atmospheric Solids Analysis Probe Quadrupole-Time of Flight Mass Spectrometry (ASAP-QTOFMS). Atmospheric Pressure Gas Chromatography Quadrupole-Time of Flight Mass Spectrometry (APGC-QTOFMS) was used to confirm the markers in different samples of oils, recycled PET (rPET), recycled cardboard and packaging of couscous and semolina to confirm the contamination. 27 markers were found in the mineral oil samples, 22 of them in $\mathrm{rPET}, 8$ in recycled board and no MOAH were found in packaging of couscous and semolina.
\end{abstract}

\section{Introduction}

Despite the large amount of testing applied to both food and food packaging materials, consumers are increasingly concerned about food safety. In-depth chemical analysis and toxicity studies often show that some families of chemicals need to be removed from the food area. This is the case of mineral oils. Once their toxicity has been demonstrated [1-5], their presence in the food context should be avoided. Mineral oils were found in food packaging and the first series of data came from the study of recycled paper and board [6,7]. Mineral oils hydrocarbons $(\mathrm{MOH})$ are mixtures of different chemical molecules derived from petroleum. Chemical analysis of mineral oils is very complex because they are very heterogeneous structures with hundreds of isomers, which may be linear alkanes, branched alkanes and multiple aromatic rings, which in turn may be alkylated and include sulfur. To facilitate the work, $\mathrm{MOH}$ are grouped into two families called MOSH and MOAH. The MOSH open chain hydrocarbons are often branched (paraffins and isoparaffins) and saturated cyclic (commonly naphthenes) hydrocarbons [8]. The individual identification is really difficult. For this reason, several approaches have been suggested by different research groups [2,9-11] However, in all cases the final quantification is done from the total area of the hump obtained after fractionation of mineral oils into the MOSH and $\mathrm{MOAH}$, and no individual confirmation of the identity is done.

The MOAH fraction depends on the composition of mineral oil and can vary between 15 and $35 \%$ and can contain mutagenic and carcinogenic activity. Recently estrogenic activity was also attributed to MOAH and probably behaviour as endocrine disruptors [12]. In 2016 the European Union published the recommendation EU 2017/84 of monitoring mineral oils in food and food packaging, with special emphasis on MOAH.

Several food products were analysed in different EU countries, pasta [13], cereals [11], dry food [14], oats or cheesecakes showed different concentrations of MOAH. In 2015, many foodstuffs were analysed in 120 countries, and $43 \%$ of the samples showed MOAH [15]. It is suggested that MOAH could come not only from mineral oils but from atmospheric pollution. However, it has been confirmed that MOAH from mineral oils are mainly those alkylated and when these alkyl derivatives are detected, about $97 \%$ are coming from mineral oils [16].

\footnotetext{
* Corresponding author.

E-mail address: cnerin@unizar.es (C. Nerín).
} 
The analysis of MOHs and MOAHs has been developed by Labor Kantonales Zurich (KLZH) and the National Reference Laboratory of Food Packaging Materials placed in Zurich [17]. The method is quite complex and involves the extraction, fractionation and analysis by HPLC-GC-FID [18,19]. Recently, they have improved the method to analyse better the MOAH using LC-GC-GC-MS, in which more information about the chemical structure of aromatic rings can be obtained [20-22]. Although the method is good for screening, it does not solve the quantitative problem, and there is still great variability in the results reported by different laboratories [16]. Recently, the Joint Research Center (JRC) prepared a packaging material with a known concentration of mineral oils to help the laboratories in this task. However, the material is not certified yet.

Without a doubt, the most difficult task in this analysis is quantification, since it is based on the humps containing the MOSH and MOAH fraction, without identifying the individual hydrocarbons under the hump. This way, several hydrocarbons different from MOAH can be coeluted, and these facts provide higher quantitative values, which will be assigned to MOAH. To eliminate this risk, some MOAH markers have been suggested, such as anthracene y perylene [23], but this is not enough. Another approach for confirming the MOAH values was the use of $m / z 91,105,113$ and 119, but these mass fragments coincide as well with many natural substances such as terpenes, phytosterols, olefins and carotenoids, what would overestimate the MOAH values [10,24]. These studies concluded that more MOAH chemical markers would be necessary to confirm the quantification of MOAH $[10,25]$. This is the main objective of the present work.

Chemical markers have the advantage of being useful tools for verifying the source of contamination and providing detailed and reliable chemical evidence of MOAH contamination, helping to avoid misinterpretations in MOAH analysis [10,16].

To find the right and useful markers for MOAH is not an easy task, as there are hundreds of aromatic hydrocarbons present in mineral oils [26-28]. Due to the complexity of mineral oils, conventional GC-MS technique is not enough to identify all potential markers and other techniques could be used. Atmospheric pressure gas chromatography coupled to high resolution MS, named APGC- MS-QTOF is a powerful technique that allows the accurate mass and thus, facilitates the identification of chemical compounds [29,30]. This technique also provides the use of $\mathrm{MS}^{\mathrm{E}}$, where all compounds are driven to the collision cell and alternatively exposed to low and high collision energy, which provides high selectivity and sensitivity.

The present work shows the study carried out to select available markers of MOAHs, which could be used to identify the contamination of MOAHs in any sample. 27 potential markers were explored, including Polycyclic Aromatic Hydrocarbons (PAHs) and their alkyl and branched derivatives as well as DBTs and BNTs isomers. 16 out of the 27 under study were finally selected as representatives of the different families present in mineral oils, in order to identify MOAH in the samples. The hyphenated techniques ASAP-QTOF-MS and APGC-QTOF-MS were used for analysing MOAH in mineral oils, recycled PET, recycled paperboard and packaging of couscous and semolina samples. The results are shown and discussed.

\section{Materials and method}

\subsection{Reagents}

The standards used were: 1,2,3-trimethylbenzene, 1,3-diethylbenzene, 2-methylnaphthalene, 1-methylnaphthalene, 4-tertbutyltoluene, biphenyl, acenaphthene, 2,6-dimethylnaphthalene, cyclohexylbenzene, fluorene, anthracene, phenanthrene, 4-methyldibenzothiophene, pyrene, 3,3',5,5'-tetramethylbiphenyl, 2,6-diisopropylnaphthalene, 4,6-dimethyldibenzothiophene, 1-methylpyrene, benz (a)anthracene, chrysene, benzo(b)naphtho(1,2-d)thiophene, 1,3,5-tritert-butylbenzene, benzo(b)fluoranthene, benzo(a)pyrene, and perylene, all supplied by were Sigma-Aldrich (Madrid, Spain). 9,9'dimethylfluorene from Tokyo Chemical Industry CO., LTD. and 3,6dimethylphenanthrene from Dr. Ehrenstorfer (Augsburg, Germany). A stock solution containing an accurate concentration of $100 \mu \mathrm{g} / \mathrm{g}$ of each compound was prepared in toluene. Lower concentrations were prepared by appropriate dilution in $n$-hexane.

The standards used for MOSH and MOAH analysis by GC-FID were nundecane, n-tridecane, bicyclohexyl, $5 \alpha$-cholestane, pentylbenzene, 1 methylnaphthalene, 2-methylnaphthalene, 1,3,5-tri-tert-butylbenzene and perylene, all acquired to Sigma-Aldrich. A solution containing: nundecane $(175 \mu \mathrm{g} / \mathrm{g}), \mathrm{n}$-tridecane $(350 \mu \mathrm{g} / \mathrm{g})$, bicyclohexyl $(350 \mu \mathrm{g} / \mathrm{g})$, $5 \alpha$-cholestane $(350 \mu \mathrm{g} / \mathrm{g})$, pentylbenzene $(350 \mu \mathrm{g} / \mathrm{g})$, 1-methylnaphthalene $(350 \mu \mathrm{g} / \mathrm{g}), 2$-methylnaphthalene $(350 \mu \mathrm{g} / \mathrm{g}), 1,3,5$-tri-tert-butylbenzene $(350 \mu \mathrm{g} / \mathrm{g})$ and perylene $(350 \mu \mathrm{g} / \mathrm{g})$ in toluene was used as internal standard. All solutions were stored at $4{ }^{\circ} \mathrm{C}$. All standards and solutions were under gravimetric control.

The solvents used were: toluene, n-hexane, methanol, dichloromethane (DCM) and acetone, all HPLC grade, supplied by Scharlab SL (Barcelona, Spain). Standard of saturated alkanes (C7-C40) of $1000 \mu \mathrm{g} /$ $\mathrm{mL}$ each component in $\mathrm{n}$-hexane was purchased from Sigma Aldrich.

Silica gel of high-purity grade with $60 \AA$ (70-230 mesh) pore size for chromatographic columns and silver nitrate on silica gel ( $\sim 10 \mathrm{wt} \%$ loading, 230 mesh) were obtained from Sigma-Aldrich (Madrid, Spain). Anhydrous sodium sulfate and cellulose extraction thimbles were purchased from Scharlab SL (Barcelona, Spain). The silanised glass wool was from Supelco (Bellefonte, USA) and ultrapure water type I (reactive grade) was obtained from Ultramatic GR water purification system (Wasserlab, Spain). Liquid nitrogen was supplied by cryogenic liquids service of Zaragoza University.

\subsection{Samples}

The mineral oil samples were hydraulic oil (oil 01), multigrade lubricant oil (oil 02), oil for rotary vane pumps and roots pumps (oil 03), lubricating oil different uses (oil 04), oil for gasoline engine (oil 05) and industrial oil use (oil 06). These samples were purchased in the local retail market. The oils chosen cover a wide range of uses in industry.

Samples of recycled polyethylene terephthalate (rPET) intended for food packaging from different companies coded as AB, FFT, TP, GE and IN were analysed. The samples of recycled cardboard were provided by a manufacturing company, and two samples of cardboard packaging in direct contact with the food, couscous and semolina, were obtained from the retail market.

\subsection{Sample preparation}

Sample extraction and treatment was different for each kind of matrix. $50 \mathrm{mg}$ of mineral oil samples were diluted to $1000 \mathrm{mg}$ with nhexane. Then a fractionation on silica impregnated with silver nitrate was applied and the MOAH fraction was separated, following the analytical method developed by KLZH and BfR [17] and recommended by JRC [24]. The mixture of silica gel with silver nitrate $(0.3 \%)$ was prepared according to the procedure described by Spack et al. [10] and homogenised for $12 \mathrm{~h}$ in a laboratory shaker Vibromatic JP Selecta (Spain).

The recycled PET pellets were cooled in liquid nitrogen and milled as powder to increase the surface and facilitate the extraction process of mineral oils. In a cellulose thimble, $35 \mathrm{~g}$ of the cryogenically powdered rPET were introduced and extracted with $\mathrm{n}$-hexane in a $250 \mathrm{~mL}$ Soxhlet for $24 \mathrm{~h}$. The extract obtained was concentrated in a rotary evaporator at $40^{\circ} \mathrm{C}$ up to $0.5 \mathrm{~g}$, and the MOAH fraction was separated using the KLZH \& BfR protocol.

Recycled cardboard samples were cut into small pieces of $0.5 \times 0.5 \mathrm{~cm}$ and $2.0 \mathrm{~g}$ exactly weighed were placed in a $20 \mathrm{~mL}$ glass vial with $10 \mathrm{~mL}$ of ethanol/n-hexane (1:1) mixture. The vial was shaken at room temperature for $2 \mathrm{~h}$ to extract the $\mathrm{MOH}$, according to the 
procedure proposed by the KLZH \& BfR. The extract was then added to a glass column containing $33 \mathrm{~g}$ of silica previously activated at $400{ }^{\circ} \mathrm{C}$ for $24 \mathrm{~h}$ in the oven and $1 \mathrm{~g}$ of silica coated with silver nitrate $10 \%(\mathrm{w} / \mathrm{w})$. MOSH and MOAH fractions were obtained using an n-hexane/ dichloromethane gradient.

All glassware was cleaned with methanol, acetone and n-hexane and dried in the oven before using. The samples analysed by ASAP-QTOF-MS did not require previous extraction or sample treatment. Blank samples were always prepared and analysed simultaneously with the samples throughout the process.

All samples were analysed in triplicate by APGC-QTOF-MS and GCFID to demonstrate the presence of contamination according to the Grob method [7].

\subsection{ASAP-QTOF-MS}

ASAP-QTOF-MS XevoG2 QTOF from Waters Corporation (Manchester, UK) was used. The instrument has a mass range up to $m / z$ 100,000 and a solving power of $>22.500$ full wide at half maximum (FWHM). Constant temperature at $120^{\circ} \mathrm{C}$ was used in the ionisation chamber. The key ion source parameters to be optimised were: corona current (mA), sample cone voltage (V) and desolvation gas temperature $\left({ }^{\circ} \mathrm{C}\right)$. Acquisition mode used was full-scan MS data from 45 to $450 \mathrm{amu}$. The working conditions were: $4 \mathrm{~min}$ of acquisition time, $650 \mathrm{~L} / \mathrm{h}$ desolvation gas, resolution mode, sampling cone 4.0, $5 \mathrm{~mA}$ crown, 4.0 cone extraction, 120 scan. Cone flow was not needed for this technique.

To facilitate the analysis of all markers in a single acquisition, a gradient of temperature from 200 to $450{ }^{\circ} \mathrm{C}$ was applied to the gas desolvation step, so that the compounds would progressively appear according to their size. To set the limit of detection (LOD) solutions of individual standards in $\mathrm{n}$-hexane were analysed at different concentrations $(0.1,0.5,1,5,10,20 \mu \mathrm{g} / \mathrm{mL})$ under the same experimental conditions as the samples. Data were collected and processed using MassLynx (Waters Corporation) software. LODs were calculated as three times the standard deviation of the blank at the same characteristic mass of each compound.

Before the analysis, the single use glass rod was inserted into the source at high temperature for $2 \mathrm{~min}$ to remove any contamination from the tip. Then it was cooled and introduced into the sample for $10 \mathrm{~s}$. After that it was inserted into the ionisation chamber of ASAP. Blanks were performed without loading the sample, during the first $120 \mathrm{~s}$ of acquisition, applying the same temperature ramp. The samples were analysed in continuous mode for $4 \mathrm{~min}$. From each MS spectrum, the characteristic masses were selected, and this information was used for searching the presence or absence of each compound in the samples. Five replicates of each sample were analysed.

\subsection{APGC-QTOF-MS}

The analysis was performed using a 7890A GC system (Agilent, Santa Clara, CA, USA) equipped with a CTC Analytics Combipal autosampler. The chromatographic separation was performed in a DB-5 MS capillary column, $30 \mathrm{~m}, 0.25 \mathrm{~mm}$ id, $0.25 \mu \mathrm{m}$ film thickness. The oven temperature program was: $40^{\circ} \mathrm{C}$ for $1 \mathrm{~min}, 7^{\circ} \mathrm{C} / \mathrm{min}$ ramp to $100^{\circ} \mathrm{C}, 9^{\circ} \mathrm{C} / \mathrm{min}$ ramp to $240^{\circ} \mathrm{C}$ and held for $1 \mathrm{~min}, 5^{\circ} \mathrm{C} / \mathrm{min}$ ramp to $300^{\circ} \mathrm{C}$ and held for $7 \mathrm{~min} .1 \mu \mathrm{L}$ was injected in splitless mode. Helium was used as carrier gas at a constant flow of $0.07 \mathrm{~L} / \mathrm{h}$.

The APGC source was coupled to a quadrupole-time of flight analyser (Q-TOF) Xevo G2 from Waters (Milford, MA, USA). API positive polarity and sensitivity analyser mode were selected. The ion source (API + mode) with a corona current of $3 \mathrm{kV}$ was used. Sampling and extraction cone voltages were $30 \mathrm{~V}$ for sampling cone and $3 \mathrm{~V}$ for extraction cone. Cone and desolvation gas flow were 20 and $175 \mathrm{~L} / \mathrm{h}$ "respectively". $\mathrm{N}_{2}$ was used as a makeup gas at $300 \mathrm{~mL} / \mathrm{min}$ and $300^{\circ} \mathrm{C}$, while the source temperature was $150^{\circ} \mathrm{C}$. Acquisition was performed in $\mathrm{MS}^{\mathrm{E}}$ mode, with collision energy of $6 \mathrm{EV}$ in function 1 (low energy), while a collision energy ramp 20-40 V was used in function 2 (high energy). Scan time was $0.5 \mathrm{~s}$, and the mass range was $45-450$ acquisition $m / z$. The lockmass reference used was perfluorotributylamine.

\subsection{GC-FID}

The GC-FID analysis was performed with a Trace GC Ultra (Thermo Electron Corporation, Milan, Italy) equipped with an AS 300
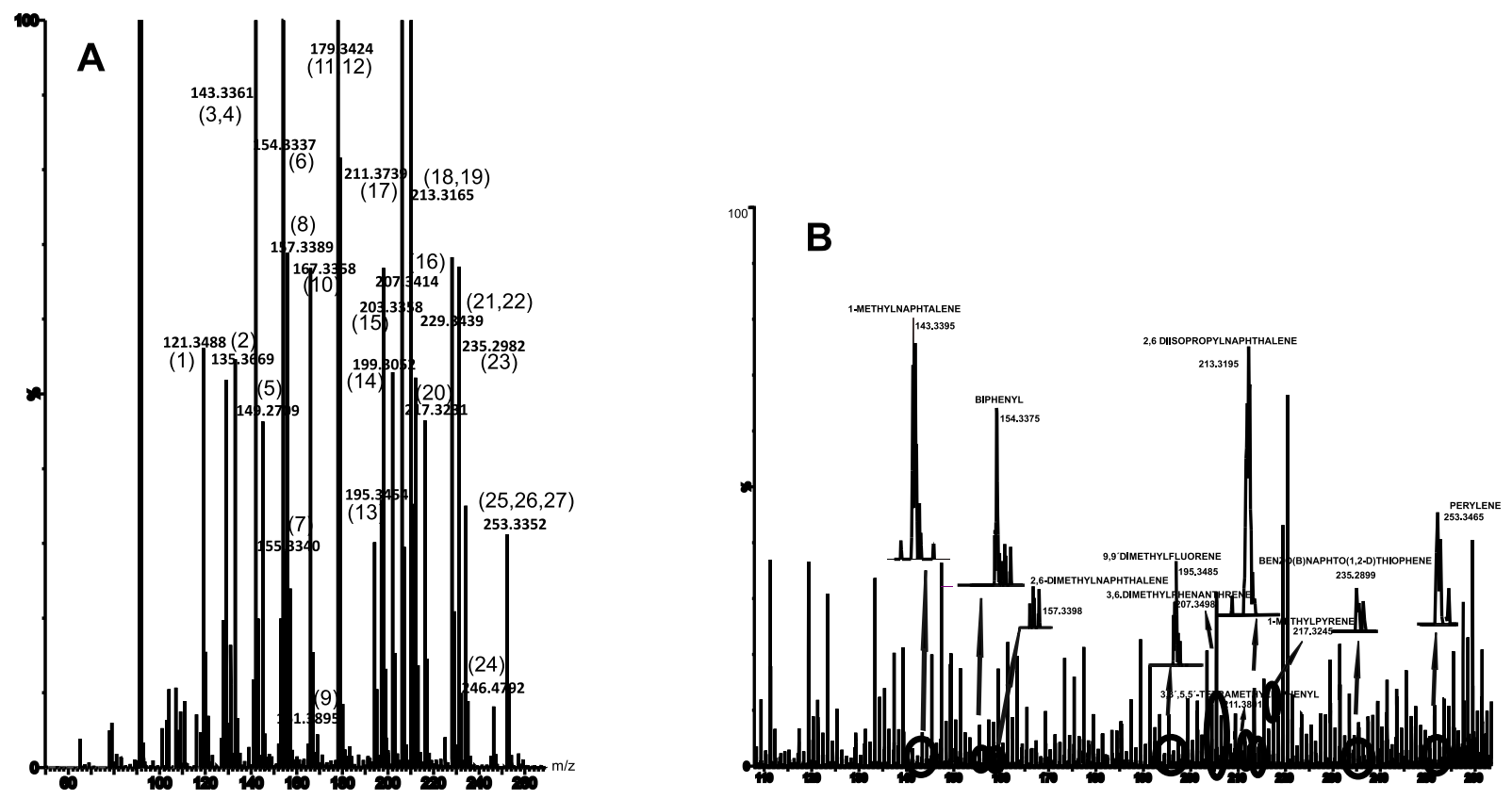

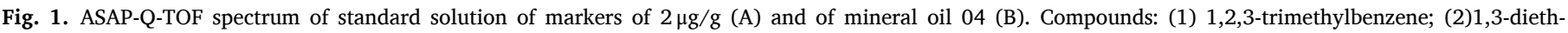

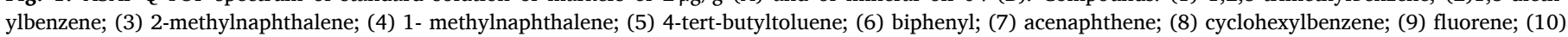

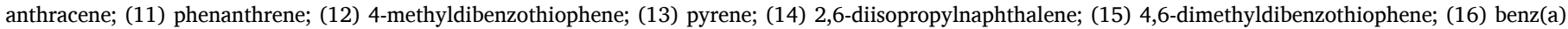

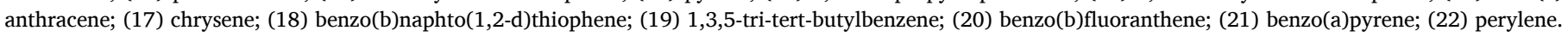




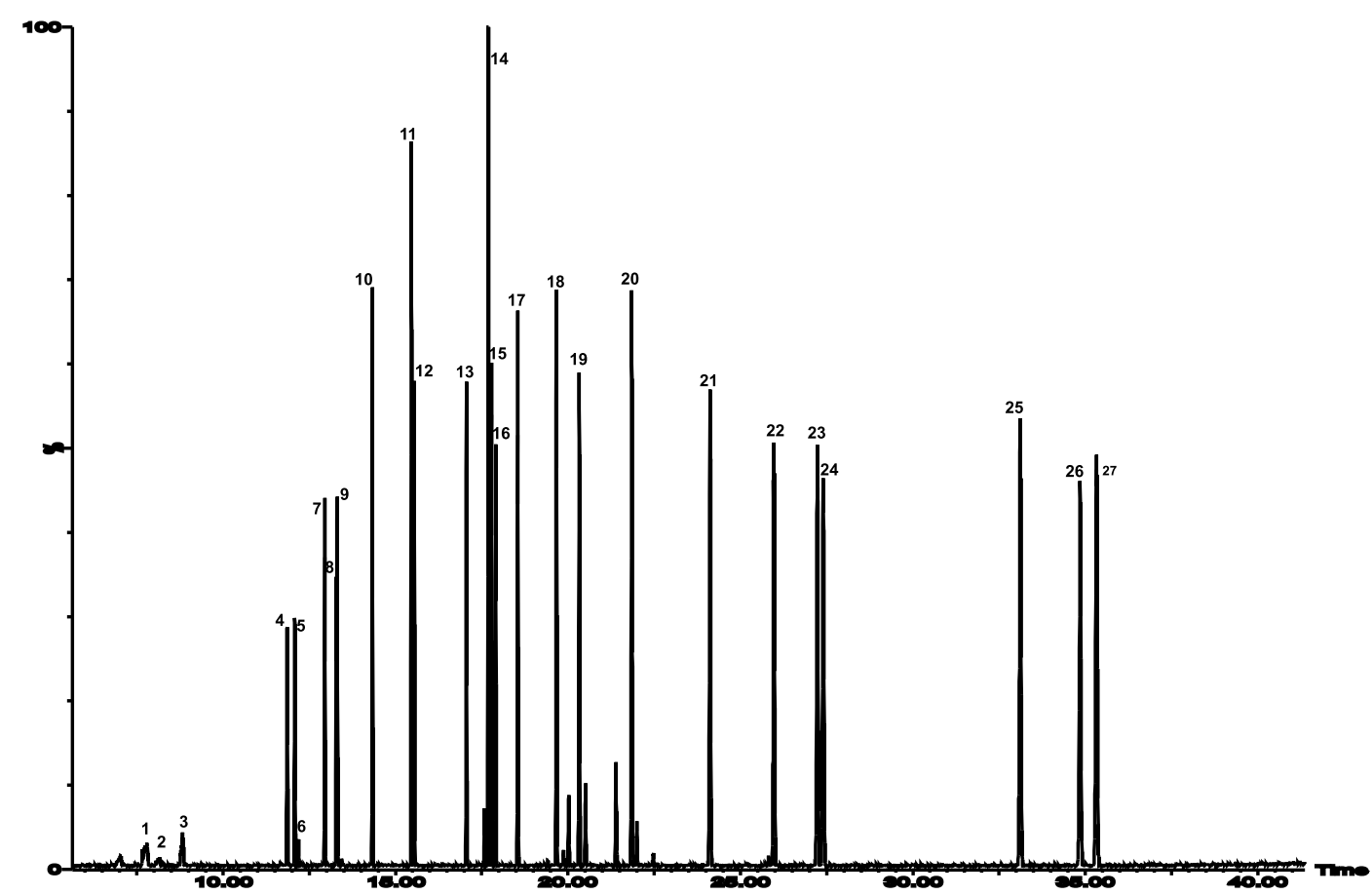

Fig. 2. Chromatogram of the standard solution ( $1 \mu \mathrm{g} / \mathrm{g}$ ) obtained by APGC-Q-TOF. Compounds: (1) 1,2,3-trimethylbenzene, (2) 1,3-diethylbenzene, (3) 4-tert-butyltoluene, (4) 2-methylnaphthalene ( $\beta$ ), (5) 1- methylnaphthalene, (6) cyclohexylbenzene, (7) biphenyl, (8) 1,3,5-tri-tert-butylbenzene, (9) 2,6-dimethylnaphthalene, (10) acenaphthene, (11) 9,9'dimethylfluorene, (12) fluorene, (13) 2,6-diisopropylnaphthalene, (14) 3,3',5,5'-tetramethylbiphenyl, (15) anthracene, (16) phenanthrene, (17) 4-methyldibenzothiophene, (18) 4,6-dimethyldibenzothiophene, (19) 3,6-dimethylphenanthrene, (20) pyrene, (21) 1-methylpyrene, (22) benzo(b) naphto [1,2-d]thiophene, (23) benz(a)anthracene, (24) chrysene, (25) benzo(b)fluranthene, (26) benzo(a)pyrene and (27) perylene.

autosampler and a flame ionisation detector (FID). The analytical column used was HP-5 (60 $\mathrm{m} \times 0.25 \mathrm{~mm}$ i.d., $0.25 \mu \mathrm{m}$ film thickness) from Agilent Technologies. The oven temperature program started at $50^{\circ} \mathrm{C}$ held for $2 \mathrm{~min}$; then increased by $30^{\circ} \mathrm{C} / \mathrm{min}$ to $310^{\circ} \mathrm{C}$ and held for $15 \mathrm{~min}$. The total run was $25.7 \mathrm{~min}$. The flow rate of the carrier gas (helium, 99.999\%) was $2.0 \mathrm{~mL} / \mathrm{min}$. Splitless injection and injector temperature $250{ }^{\circ} \mathrm{C}$ were used. The injection volume was $5 \mu \mathrm{L}$. The FID detector temperature was $350^{\circ} \mathrm{C}$. Data were acquired and processed with Chrom-Card GC Software (Thermo Electron).

\section{Results and discussion}

\subsection{Direct identification of markers of mineral oils by ASAP-MS-Q-TOF}

The most complex and expensive step of any analysis is the sample treatment, and mineral oils are very difficult samples in which this step is tedious and time consuming. The major advantage of ASAP is that it allows the direct analysis without sample pretreatment and can be applied to targeted volatile or semi-volatile compounds. The presence or absence of specific compounds can be quickly determined by comparing their exact mass with that of a pure standard. As another technique, ASAP needs the optimisation of certain key parameters, such as the corona current (A), sample cone voltage (V) and desolvation gas temperature $\left({ }^{\circ} \mathrm{C}\right)$. These parameters will influence the total number of ions reaching the detector.

The first step was to optimise the experimental conditions for the selected standard compounds. The key parameters above mentioned were optimised using standard solutions of the pure compounds. Cone voltage sampling varied from $20 \mathrm{~V}$ to $80 \mathrm{~V}$ and the cone voltage extraction was set at $0.1 \mathrm{~V}$. Target samples were analysed in continuous mode ( $3 \mathrm{~min}$ ) with a cone voltage ramp (20-80 V) and desolvation gas temperature ramp $\left(200-500{ }^{\circ} \mathrm{C}\right)$ Atmospheric Pressure Ionisation (API) in positive polarity was selected, and source temperature was $120^{\circ} \mathrm{C}$. The parameters of the XEVO G2 QTOF were: scan time $1 \mathrm{~s}$ and the mass range considered was $m / z 45-450$ to ensure the presence of all standards. Each sample was analysed in triplicate. A blank sample was also analysed under the same experimental conditions. In addition to the high resolution mass achieved, isotopic ratios (C12/C13, N14/N15, O16/O18) and software tools were used to confirm the target compounds. MassLynx software from Waters was used, which considers the isotopic model and the elemental composition. From the mass spectrum of each standard, the characteristic masses were selected and later used to determine the presence or absence of the compound in the sample.

Table S1 shows the results of all analysed oils with their molecular structure, molecular weight, and CAS number of the standards. As can be seen, twelve compounds 1,2,3-trimethylbenzene, biphenyl, 2,6-dimethylnaphthalene, anthracene, 9,9'-dimethylfluorene, 4-methyldibenzothiophene, pyrene, 3,6-dimethylphenanthrene, 3,3',5,5'tetramethylbiphenyl, benz(a)anthracene, 1-methylpyrene and perylene, were detected in all oil samples. Thus, these compounds could be used as markers of aromatic mineral oils, as they take part in the composition of different types of oils [27,28,31-33].

Fig. 1 A shows the spectrum of a mixture of potential markers of $2 \mu \mathrm{g} / \mathrm{g}$ of MOAH analysed by ASAP-QTOF-MS in positive mode. The exact mass allows to check the presence or absence of these compounds in the obtained spectra of mineral oils, with an error lower than $0.5 \mathrm{Da}$ as shows Fig. 1 A. The molecular ion $[\mathrm{M}]^{+}$and protonated ion $[\mathrm{MH}]^{+}$were the most abundant ions in all compounds. This performance was also observed in previous work [34]. LOD was found to determine the order of magnitude in which the markers could be detected in the samples.

Direct analysis provides the exact masses of all compounds present in the sample but without chromatographic separation, isobaric isomers, such as 2-methylnaphtalene and 1-methylnaphtalene isomers, or biphenyl and acenaphthene among others, which have exactly the same $\mathrm{m} / \mathrm{z}$ ratio, cannot be unequivocally identified.

Fig. 1 B shows the MS spectrum of oil 04, where the masses of 1methylnaphthalene, biphenyl, 2.6 dimethylnaphthalene, 9,9-dimethylfluorene, 3,6-dimethylphenanthrene, 3,3',5,5'-tetramethylbiphenyl, 
Table 1

Compounds identified in mineral oil and/or paperboard samples.

\begin{tabular}{|c|c|c|c|c|c|c|c|c|}
\hline \multirow[t]{2}{*}{ COMPOUNDS } & \multicolumn{5}{|c|}{ SAMPLES PET } & \multicolumn{3}{|c|}{ SAMPLES PAPERBOARD } \\
\hline & $\mathrm{AB}$ & FFT & $\mathrm{TP}$ & $\mathrm{GE}$ & IN & RECYCLED & couscous & SEMOLINA \\
\hline 1,2,3-Trimethylbenzene & $\checkmark$ & $\checkmark$ & $\checkmark$ & $\checkmark$ & & $\checkmark$ & $\checkmark$ & $\checkmark$ \\
\hline 1,3-Diethylbenzene & $\checkmark$ & $\checkmark$ & $\checkmark$ & $\checkmark$ & $\checkmark$ & $\checkmark$ & $\checkmark$ & $\checkmark$ \\
\hline 4-tert-Butyltoluene & & & & & $\checkmark$ & $\checkmark$ & $\checkmark$ & $\checkmark$ \\
\hline 2-Methylnaphthalene ( $\beta$ ) & $\checkmark$ & $\checkmark$ & $\checkmark$ & $\checkmark$ & $\checkmark$ & & & \\
\hline 1- Methylnaphthalene & $\checkmark$ & $\checkmark$ & $\checkmark$ & & & & & \\
\hline Cyclohexylbenzene & $\checkmark$ & $\checkmark$ & & $\checkmark$ & & & & \\
\hline Biphenyl & & $\checkmark$ & $\checkmark$ & $\checkmark$ & $\checkmark$ & & & \\
\hline 1,3,5-Tert-butylbenzene & $\checkmark$ & & & & & & & \\
\hline 2,6-Dimethylnaphthalene & $\checkmark$ & $\checkmark$ & $\checkmark$ & $\checkmark$ & $\checkmark$ & & & \\
\hline Acenaphthene & $\checkmark$ & $\checkmark$ & $\checkmark$ & & & & & \\
\hline 9,9'Dimethylfluorene & & $\checkmark$ & $\checkmark$ & $\checkmark$ & $\checkmark$ & $\checkmark$ & & \\
\hline Fluorene & & $\checkmark$ & $\checkmark$ & & $\checkmark$ & & & \\
\hline 2,6-Diisopropylnaphthalene & $\checkmark$ & $\checkmark$ & $\checkmark$ & $\checkmark$ & $\checkmark$ & $\checkmark$ & & \\
\hline $3,3^{\prime}, 5,5^{\prime}$-Tetramethylbiphenyl & & $\checkmark$ & & $\checkmark$ & $\checkmark$ & & & \\
\hline Anthracene & $\checkmark$ & $\checkmark$ & & & & & & \\
\hline Phenanthrene & $\checkmark$ & $\checkmark$ & & & & & & \\
\hline 4-Methyldibenzothiophene & $\checkmark$ & $\checkmark$ & $\checkmark$ & $\checkmark$ & & & & \\
\hline 4,6-Dimethyldibenzothiophene & $\checkmark$ & $\checkmark$ & $\checkmark$ & $\checkmark$ & $\checkmark$ & & & \\
\hline 3,6-Dimethylphenanthrene & $\checkmark$ & $\checkmark$ & $\checkmark$ & $\checkmark$ & & $\checkmark$ & & \\
\hline Pyrene & $\checkmark$ & $\checkmark$ & $\checkmark$ & $\checkmark$ & $\checkmark$ & & & \\
\hline 1-Methylpyrene & $\checkmark$ & $\checkmark$ & & $\checkmark$ & $\checkmark$ & $\checkmark$ & & \\
\hline Benzo(b)naphto[1,2-d]thiophene & $\checkmark$ & $\checkmark$ & & & & & & \\
\hline Benz(a)anthracene & $\checkmark$ & & & & & & & \\
\hline Chrysene & $\checkmark$ & & $\checkmark$ & $\checkmark$ & $\checkmark$ & & & \\
\hline Benzo(b)fluoranthene & $\checkmark$ & & & & & & & \\
\hline Benzo(a)pyrene & $\checkmark$ & $\checkmark$ & $\checkmark$ & & & & & \\
\hline Perylene & $\checkmark$ & $\checkmark$ & & & & & & \\
\hline
\end{tabular}

2,6-diisopropylnaphthalene, 1-methylpyrene, benzo(b)naphtho(1,2-d) thiophene and perylene, can be seen. Fluorene, dibenzothiophene and dibenzofuran and their alkylated homologs are the most important aromatic compounds in crude oils.

Although ASAP was an excellent tool for fast screening of MOAH in difficult samples, some of the common isomers could not be properly identified and additional in-depth analysis using a chromatographic separation was required.

\subsection{Markers analysis by APGC-Q-TOF-MS}

Once a set of 12 markers were initially identified, the objective was to detect them in different food packaging materials. The selected samples were recycled PET, recycled cardboard and two samples of cardboard packaging of couscous and semolina from the retail market.

As was above mentioned, one of the problems in ASAP is the inability to differentiate the isomers without prior separation. This problem is solved with chromatography. However, the usual GC-MS analysis of mineral oils provides a forest of peaks that make almost impossible the identification of individual compounds and additional tools are required for this purpose. Using QTOF analyser and $\mathrm{MS}^{\mathrm{E}}$ mode, the unequivocal identification of MOAH in the samples was achieved. Data for accurate molecular mass ions were processed using MassLynx, a software program that provides the corresponding elemental composition and the difference between experimental and theoretical mass. It was also necessary to optimise the chromatographic conditions to achieve a good separation of all standards including the isomers. Fig. 2 shows the chromatogram obtained of a mixture of standards $3 \mu \mathrm{g} / \mathrm{g}$ in $\mathrm{n}$-hexane. As can be seen, the isomers: 2-methylnaphthalene and 1-methylnaphthalene $(4,5)$ with molecular mass 142.20 ; biphenyl and acenaphthene $(7,10)$ with molecular mass 154.21 ; anthracene and phenanthrene $(15,16)$ with more molecular mass 178.23; 2,6-diisopropylnaphthalene and 4,6-dimethyldibenzothiophene $(13,18)$ with molecular mass 212.31 , benz(a)anthracene and chrysene $(23,24)$ with molecular mass 228.29 and the three isomers benzo(b)fluoranthene, benzo(a)pyrene and perylene $(25,26,27)$ with molecular weight 252.31 were perfectly separated by chromatography.
The spectra of standards showed gently ionized analytes with low or any fragmentation. The most abundant compounds were protonated $[\mathrm{M}+\mathrm{H}]^{+}$and deprotonated $[\mathrm{M}-\mathrm{H}]^{-}$, in addition to some methylated $\left[\mathrm{M}-\mathrm{CH}_{3}\right]^{+}$. The ionisation process with APGC can be driven towards protonation by using modifiers within the source enclosure, typically $\mathrm{H}_{2} \mathrm{O}$ or $\mathrm{MeOH}$. The modifier is simply a vial of reagent which is located within either the source door (cool position) or ion block holder (heated position). Individual standard solutions in $\mathrm{n}$-hexane at different concentrations $(0.01,0.05,0.1,0.5,1,5,10,20 \mu \mathrm{g} / \mathrm{g})$ were analysed to determine the analytical features. Table S2 shows the characteristics of the method with retention times, characteristic mass, LOD of the compounds as well as the reproducibility and accuracy. LOD was calculated as three times the standard deviation "respectively" of the blank at the same characteristic mass of each compound. All LOD values were in the range of $0.01-0.06 \mu \mathrm{g} / \mathrm{g}$.

To evaluate the reproducibility, repeatability and accuracy, the samples were analysed five times in the same day (intra-day precision) and eleven times in five different days (interday precision) and the resulting relative standard deviation of five injections were calculated. The accuracy values vary from 1.1 to 5.6 for intra-day and from 1.9 to 11.2 for interday with relative standard deviation between 5.6 and $11.2 \%(n=5)$, being the highest value to perylene. These parameters were used to identify the markers in the samples.

\subsection{Identification of markers in samples by APGC-Q-TOF-MS}

The identification was performed using the software ChromaLynx XS (SCN 714) in both targeted and non-targeted mode. For the targeted mode, a database library containing the $27 \mathrm{MOAH}$ species, which were previously identified by GC-MS, was built and used for the purpose.

The accurate theoretical masses of the compounds $[\mathrm{M}]^{+}$, protonated compounds $[\mathrm{M}+\mathrm{H}]^{+}$and deprotonated $[\mathrm{M}-\mathrm{H}]^{-}$were taken into account for building the database.

The chromatograms were processed with ChromaLynx, with a narrow window of mass $( \pm 15 \mathrm{mDa})$ and the retention time window of $\pm 0.20 \mathrm{~min}$, to confirm the presence or the absence of the analytes of interest. Based on these data and the comparison with those obtained 


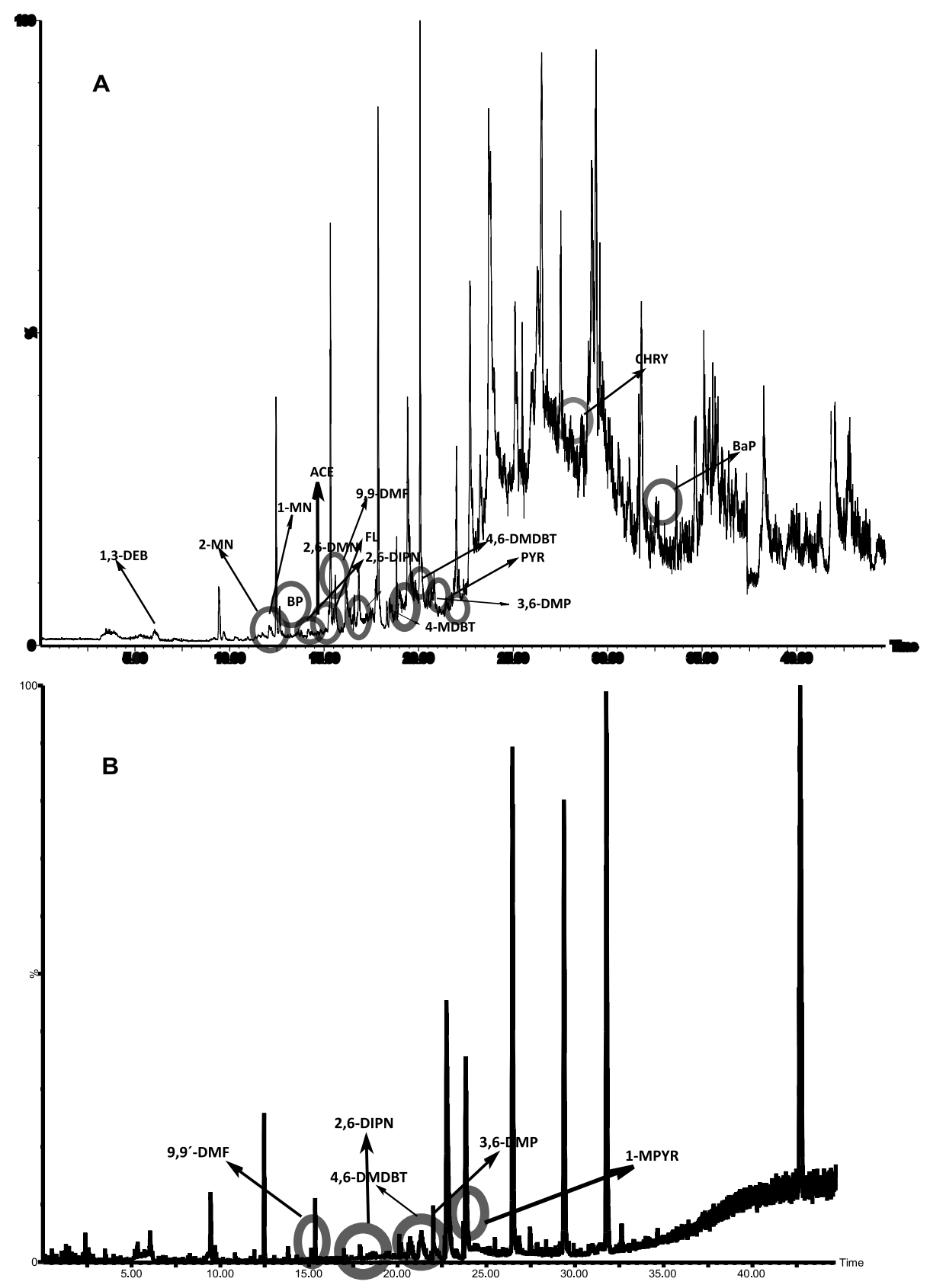

Fig. 3. Chromatograms obtained by APGC-Q-TOF for rPET TP (a) and the paperboard (b) samples.

with standards, the results were confirmed in the samples.

When concentrations were very low, and there were plenty of low intensity ions, the deconvolution of the mass spectrum for each compound under study helped us to confirm the identification. The results of the presence of MOAH found in the samples, after analysing four replicates of each sample, are shown in Table 1.

Five samples of rPET, one recycled cardboard and two samples of food contact packaging, couscous and semolina were analysed. Fig. $3 \mathrm{~A}$ shows the chromatogram with the identified compounds in the rPET sample coded as TP. Fig. $3 \mathrm{~B}$ shows the chromatogram of the recycled cardboard with the compounds identified.

22 out of the 27 selected markers were found in $\mathrm{PPET}$ AB and FFT samples. In rPET TP sample 17 markers were found, 15 markers were found in rPET GE sample and 14 markers in rPET IN.

PET AB and FFT samples were the most contaminated ones and in which more compounds were identified. Some of the markers, such as the alkylated aromatic 1, 2, 3-trimethylbenzene and 1,3-diethylbenzene, were common to all rPET samples. Among the MNs family, 2-methylnaphthalene was found in all rPET samples and its isomer 1-methylnaphthalene in three samples. As marker of MDBTs family, 4methyldibenzothiophene was found in all samples except rPET IN and as marker of BNTs family, benzo(b)naphto(1,2-d)thiophene was only found in samples of rPET AB and rPET FFT. Biphenyl was found in all samples of rPET with the only exception of rPET AB sample.

2,6-dimethylnaphthalene and alkylated PAHs appeared in all rPET samples; 9,9'-dimethylfluorene was present in all rPET samples except $\mathrm{AB}$; 3,6-dimethylphenanthrene appeared in all rPET samples except IN and 1-methylpyrene was found in rPET samples except TP.

4,6-dimethyldibenzothiophene considered one of the most abundant compounds in MOAH [35] and suggested by Spack et al. [10] to identify and characterize mineral oils, was identified in all rPET samples. After carbon and hydrogen, sulfur is considered the most important element in crude oils. Thiophene joined to polycyclic aromatic hydrocarbons form sulfur heterocycles and leads to different families, including 

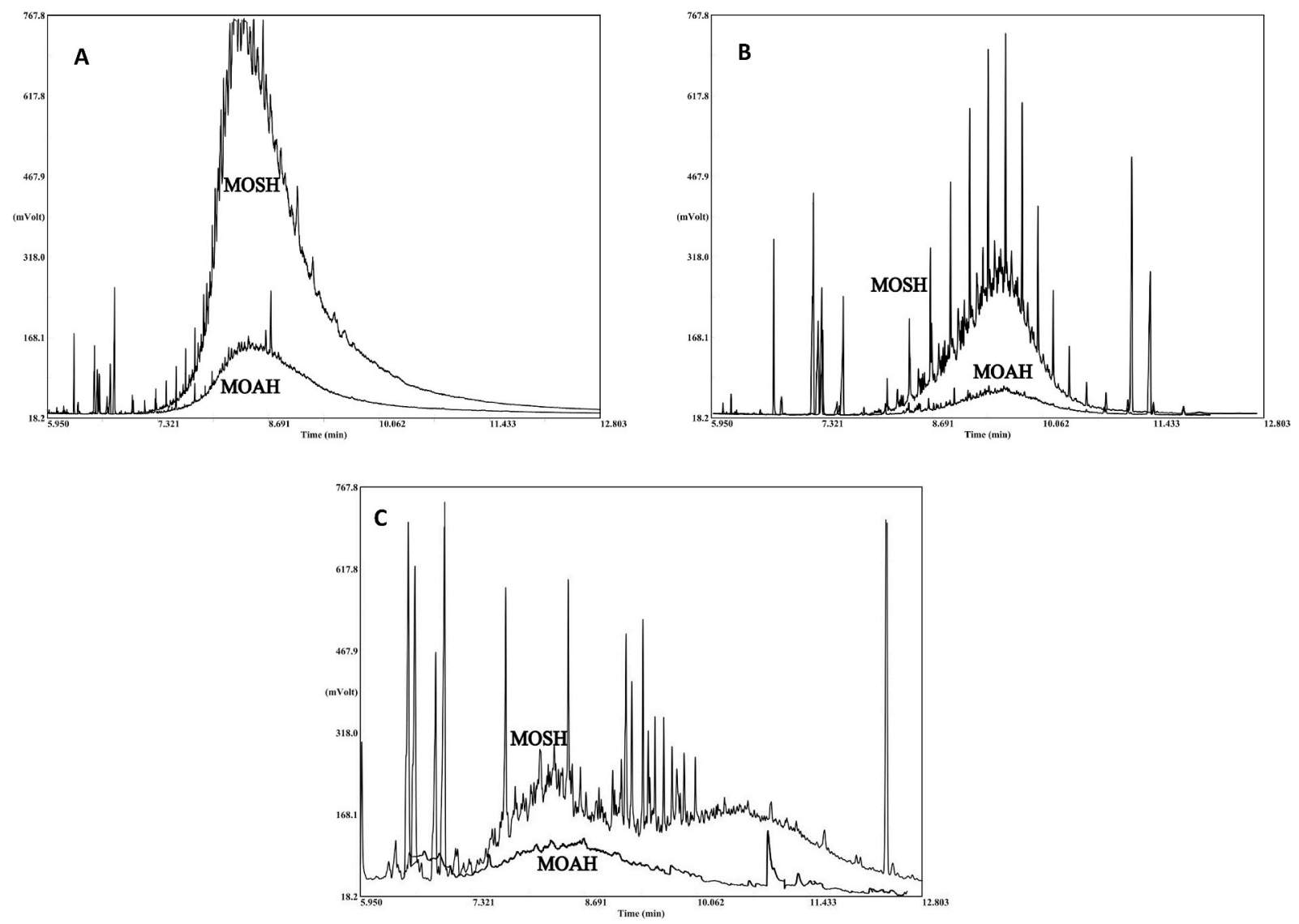

Fig. 4. Chromatogram of the mineral oil 2 sample obtained by GC-FID (A), chromatogram of the PET TP sample obtained by GC-FID (B) and chromatogram of the paperboard sample obtained by GC-FID (C).

benzothiophene (BT), dibenzothiophene (DBT), benzonaphthothiophenes (BNT) and their alkylated homologous series [27,36,37].

In the recycled cardboard sample only 1,2,3-trimethylbenzene, 1,3diethylbenzene, 4-tert-butyltoluene, acenaphthene, DIPN, 3,6-dimethylphenanthrene, 1-methylpyrene and 4,6-dimethyldibenzothiophene were found. DIPN is characteristic of recycled board [10].

Only 1,2,3-trimethylbenzene, 1,3-diethylbenzene and 4-tert-butyltoluene were detected in food-contact packaging samples (couscous and semolina), but no other MOAH markers of those studied that could demonstrate MOAH contamination in these packaging were found. 1,3,5-tri-tert-butylbenzene was barely present in the samples under study.

The semi-quantitative estimation of the results obtained for the individual markers is in the order of $0.05 \mu \mathrm{g} / \mathrm{g}$ benz(a)anthracene at $0.15 \mu \mathrm{g} / \mathrm{g}$ 4,6-dimethyldibenzothiophene for $\mathrm{rPET} \mathrm{AB}, 0.07 \mu \mathrm{g} / \mathrm{g}$ for benzo(b)naphto[1,2-d] thiophene at $0.12 \mu \mathrm{g} / \mathrm{g}$ 2,6-diisopropylnaphthalene for rPET FFT, $0.05 \mu \mathrm{g} / \mathrm{g}$ pyrene at $0.11 \mu \mathrm{g} / \mathrm{g}$ biphenyl for rPET $\mathrm{TP}, 0.06 \mu \mathrm{g} / \mathrm{g}$ chrysene at $0.09 \mu \mathrm{g} / \mathrm{g}$ 2,6-diisopropylnaphthalene for rPET GE, $0.06 \mu \mathrm{g} / \mathrm{g}$ 4,6-dimethyldibenzothiophene at $0.09 \mu \mathrm{g} / \mathrm{g}$ biphenyl for $\mathrm{rPET}$ IN and $0.06 \mu \mathrm{g} / \mathrm{g}$ 2,6-diisopropylnaphthalene at $0.11 \mu \mathrm{g} / \mathrm{g}$ 3,6-dimethylphenanthrene for recycled paperboard.

From these results it is clear that APGC-Q-TOF-MS identified without a doubt the presence of specific markers of MOAH, which demonstrates the contamination of MOAH in the samples of rPET and cardboard under study.

\subsection{Confirmation of mineral oils contamination by GC-FID}

All samples were treated according to the BfR \& KLZH protocol and analysed by GC-FID to verify the formation of the characteristic humps of the oils and to know the distribution range of their molecular masses.
The characterisation of the molecular mass of the mineral oil was carried out, overlapping the MOSH and MOAH chromatograms obtained by GC-FID and a C7-C40 saturated alkane standard, injected under the same chromatographic conditions as the samples, at concentration $5 \mu \mathrm{g} /$ g.

In the case of pure mineral oil samples, both MOSH and MOAH signals ranged from n-C-13 to n-C35 (see Fig. 4 A). On the other hand, the rPET samples, analysed during the present investigation, showed the presence of typical chromatographic humps of MOSH and MOAH fractions of mineral oil (see Fig. 4 B) with a molecular mass distribution for MOSH and MOAH between n-C14 and n-C25, which corresponds to the hydrocarbon mass range, volatile enough to migrate through the gas phase at room temperature. It should be noted that at higher temperatures, higher molecular weight hydrocarbons can also migrate. The mineral oil chromatograms of the recycled cardboard (see Fig. 4C) ranged from n-C14 to n-C28, thus indicating contamination with heavier mineral oils. The range found matches that found in mineral oil samples [38]. The MOAH content in rPET ranged from 8.62 to $16.33 \mathrm{mg} / \mathrm{kg}$, and for recycled cardboard, it was $25.12 \mathrm{mg} / \mathrm{kg}$. The RSD of the samples was less than $10 \%$.

\subsection{Final selection of $M O A H$ markers}

To determine without any doubt if MOAH are present in any sample, identification of MOAH markers is a useful way, as it will avoid misidentification due to the lack of knowledge of what is present under the hump.

Markers such as alkylated naphthalene, phenanthrene, dibenzothiophene, fluorene, and chrysene were previously used to determine mineral oils [39]. Other studies used naphthalene, 1-methylnaphthalene, 1-ethylnaphthalene, acenaphthylene, acenaphthene, 2,3, 
6-trimethylnaphthalene, fluorene, phenanthrene, 2-methylphenanthrene, 1-methylphenanthrene, 3,6-dimethylphenanthrene, fluoranthene, pyrene, 1-methylpyrene, anthracene, chrysene and perylene [40] as markers. However, most of them are also common to air pollution and the influence of mineral oils cannot be properly distinguished. Based on the results obtained in this study, a series of $16 \mathrm{MOAH}$ markers can be proposed as representatives of the different families present in mineral oils. These markers are the following ones:

PAHs: biphenyl, acenaphthene, benzo(b)fluoranthene, chrysene and perylene.

Alkylated PAHs: 2,6-dimehtylnaphthalene, 9,9'-dimethylfluorene, $3,3^{\prime}, 5,5^{\prime}$-tetramethylbiphenyl and 1-methylpyrene.

Methylnaphthalenes (MNs): 2-methylnaphthalene and 1methylnaphthalene.

MDBTs: 4-methyldibenzothiophene.

DIPNs: 2,6-diisopropylnaphthalene.

DMDBTs: 4,6-dimethyldibenzothiophene.

BNTs: benzo(b)naphto(1,2-d)thiophene.

They cover different families of mineral oils and represent the most toxic compounds. Consequently, they can be considered the most important ones to detect contamination by MOAH in any sample.

\section{Conclusions}

This study investigated the application of two techniques, ASAPQTOF-MS and APGC-QTOF-MS, to select the specific markers of MOAH present in food packaging material. Working with these selective techniques possible confusion with the presence of other substances is avoided and clear identification of MOAH was achieved.

The selection of markers was firstly done by direct targeted analysis of several mineral oils by ASAP-QTOF-MS, in which the exact mass of potential MOAH was used. In the six mineral oils of different origins, the presence of most of these markers was verified. $16 \mathrm{MOAH}$ markers out of 27 under study were selected to be used as tracers of possible mineral oil contamination.

Using APGC-QTOF-MS, a method was developed with the best chromatographic conditions for the analysis of the markers under study. The appropriate sample treatment was optimised to verify the presence of some of them in a series of rPET samples, recycled cardboard and food packaging. This contamination was also confirmed by the GC-FID method proposed by Grob.

Detection of these markers undoubtedly determines the presence of traces of MOAH contamination in food packaging.

\section{Author statement}

Janira Jaén: Methodology, Validation, Investigation, Data curation, Writing - original draft; Conceptualization, Formal analysis, Writing review \& editing, Celia Domenio: Conceptualization, Methodology, Validation, Investigation, Data curation, Writing - original draft Writing - Review \& Editing, Supervision; Pilar Alfaro: Methodology, Investigation, Data curation; Cristina Nerin: Conceptualization, Resources, Writing - review \& editing, Supervision, Project administration, Funding acquisition.

\section{Declaration of competing interest}

The authors declare that they have no known competing financial interests or personal relationships that could have appeared to influence the work reported in this paper.

\section{Acknowledgements}

This work has been financed by Secretaria Nacional de Ciencia, Tecnología e Innovación de Panamá (SENACYT), Universidad de Panamá, Gobierno de Aragón and Fondo Social Europeo (Spain) through the
GUIA Group T53_20R Grupo Consolidado de Investigación.

\section{Appendix A. Supplementary data}

Supplementary data to this article can be found online at https://doi. org/10.1016/j.talanta.2020.122079.

\section{References}

[1] IARC, Mineral oils, untreated or mildly treated, Monographs on the Evaluation of Carcinogenic Risks to Humans 100 (2012) 179-196.

[2] K. Grob, Toxicological assessment of mineral hydrocarbons in foods: state of present discussions, J. Agric. Food Chem. 66 (2018) 6968-6974, https://doi.org/ 10.1021/acs.jafc.8b02225.

[3] R. Pirow, A. Blume, N. Hellwig, M. Herzler, B. Huhse, C. Hutzler, K. Pfaff, H. J. Thierse, T. Tralau, B. Vieth, A. Luch, Mineral oil in food, cosmetic products, and in products regulated by other legislations, Crit. Rev. Toxicol. 49 (2019) 742-789, https://doi.org/10.1080/10408444.2019.1694862.

[4] H. Buist, T. van Harmelen, C. van den Berg, W. Leeman, M. Meima, L. Krul, Evaluation of measures to mitigate mineral oil migration from recycled paper in food packaging, Packag. Technol. Sci. (2020) 1-16, https://doi.org/10.1002/ pts.2534.

[5] T. Gude, Properties of typical products requiring safety assessments: focus on nonintentionally added substances (NIAS), Toxicol. Lett. (2019) 314. S7-S7.

[6] K. Grob, M. Lanfranchi, J. Egli, A. Artho, Determination of food contamination by mineral-oil from jute sacks using coupled LC-GC, J. Assoc. Off. Anal. Chem. 74 (1991) 506-512.

[7] K. Grob, M. Huber, U. Boderius, M. Bronz, Mineral oil material in canned foods, Food Addit. Contam. 14 (1997) 83-88.

[8] European Food Safety Authority (EFSA), Scientific opinion on mineral oil hydrocarbons in food, EFSA Journal (2012), https://doi.org/10.2903/j. efsa.2012.2704.

[9] A. Vollmer, M. Biedermann, F. Grundböck, J.E. Ingenhoff, S. Biedermann-Brem, W. Altkofer, K. Grob, Migration of mineral oil from printed paperboard into dry foods: survey of the German market, Eur. Food Res. Technol. 232 (2011) 175-182, https://doi.org/10.1007/s00217-010-1376-6.

[10] L.W. Spack, G. Leszczyk, J. Varela, H. Simian, T. Gude, R.H. Stadler, Understanding the contamination of food with mineral oil: the need for a confirmatory analytical and procedural approach, Food Addit. Contam. Part A Chemistry, Analysis, Control, Exposure and Risk Assessment 34 (2017) 1052-1071, https://doi.org/ 10.1080/19440049.2017.1306655.

[11] S. Moret, M. Scolaro, L. Barp, G. Purcaro, L.S. Conte, Microwave assisted saponification (MAS) followed by on-line liquid chromatography (LC)-gas chromatography (GC) for high-throughput and high-sensitivity determination of mineral oil in different cereal-based foodstuffs, Food Chem. 196 (2016) 50-57, https://doi.org/10.1016/j.foodchem.2015.09.032.

[12] P. Tarnow, C. Hutzler, S. Grabiger, K. Schön, T. Tralau, A. Luch, Estrogenic activity of mineral oil aromatic hydrocarbons used in printing inks, PloS One 11 (2016) 1-15, https://doi.org/10.1371/journal.pone.0147239.

[13] L. Barp, M. Suman, F. Lambertini, S. Moret, Migration of selected hydrocarbon contaminants into dry pasta packaged in direct contact with recycled paperboard, Food Addit. Contam. Part A Chemistry, Analysis, Control, Exposure and Risk Assessment 32 (2015) 271-283, https://doi.org/10.1080/19440049.2014.999259.

[14] S. Moret, M. Scolaro, L. Barp, G. Purcaro, M. Sander, L.S. Conte, Optimisation of pressurised liquid extraction (PLE) for rapid and efficient extraction of superficial and total mineral oil contamination from dry foods, Food Chem. 157 (2014) 470-475, https://doi.org/10.1016/j.foodchem.2014.02.071.

[15] F.P. Forum, Dossier - Mineral Oil Hydrocarbons, 2017, pp. 1-8, https://doi.org/ 10.5281/zenodo.820984.

[16] S. Koster, J. Varela, R.H. Stadler, J. Moulin, C. Cruz-Hernandez, J. Hielscher, C. Lesueur, J. Roïz, H. Simian, Mineral oil hydrocarbons in foods: is the data reliable? Food Addit. Contam. Part A Chemistry, Analysis, Control, Exposure and Risk Assessment 37 (2020) 69-83, https://doi.org/10.1080/ 19440049.2019.1678770.

[17] Bundesinstitut für Risikobewertung (BfR), Kantonales Labor Zurich (KLZH), Determination of Hydrocarbons from Mineral Oil (MOSH \& MOAH) or Plastics (POSH \& PAO) in Packaging Materials and Dry Foodstuffs by Solid Phase Extraction and GC-FID, 2011.

[18] M. Biedermann, K. Grob, On-line coupled high performance liquid chromatography-gas chromatography for the analysis of contamination by mineral oil. Part 2: migration from paperboard into dry foods: interpretation of chromatograms, J. Chromatogr. A 1255 (2012) 76-99, https://doi.org/10.1016/j. chroma.2012.05.096.

[19] L. Barp, G. Purcaro, S. Moret, L.S. Conte, A high-sample-throughput LC-GC method for mineral oil determination, J. Separ. Sci. 36 (2013) 3135-3139, https://doi.org/ 10.1002/jssc.201300114.

[20] K. Fiselier, F. Grundböck, K. Schön, O. Kappenstein, K. Pfaff, C. Hutzler, A. Luch, K. Grob, Development of a manual method for the determination of mineral oil in foods and paperboard, J. Chromatogr. A 1271 (2013) 192-200, https://doi.org/ 10.1016/j.chroma.2012.11.034.

[21] M. Biedermann, K. Grob, Comprehensive two-dimensional gas chromatography for characterizing mineral oils in foods and distinguishing them from synthetic 
hydrocarbons, J. Chromatogr. A 1375 (2015) 146-153, https://doi.org/10.1016/j. chroma.2014.11.064.

[22] M. Biedermann, K. Grob, Comprehensive two-dimensional GC after HPLC preseparation for the characterization of aromatic hydrocarbons of mineral oil origin in contaminated sunflower oil, J. Separ. Sci. 32 (2009) 3726-3737, https:// doi.org/10.1002/jssc.200900366.

[23] C. Maes, G.M. Yuki, C. Teniers, W. Luyten, G. Herremans, R. Peeters, P. Samyn, R. Carleer, M. Buntinx, Ethylene vinyl alcohol copolymer (EVOH) as a functional barrier against surrogate components migrating from paperboard, J. Chem. (2019) (2019) 7, https://doi.org/10.1155/2019/4180708. Article ID 4180708.

[24] JRC115694, Guidance on sampling, analysis and data reporting for the monitoring of mineral oil hydrocarbons in food and food contact materials, in: The Frame of Commission Recommendation (EU) 2017/84, 2019, https://doi.org/10.2760/ 208879.

[25] M. Biedermann, C. Munoz, K. Grob, Update of on-line coupled liquid chromatography - gas chromatography for the analysis of mineral oil hydrocarbons in foods and cosmetics, J. Chromatogr. A 1521 (2017) 140-149, https://doi.org/10.1016/j.chroma.2017.09.028.

[26] M.E. MacHado, E.W. De Menezes, L.P. Bregles, E.B. Caramão, E.V. Benvenutti, C A. Zini, Palladium(II) chemically bonded to silica surface applied to the separation and identification of polycyclic aromatic sulfur heterocycles in heavy oil, J. Separ. Sci. 36 (2013) 1636-1643, https://doi.org/10.1002/jssc.201200773.

[27] M. Li, T.G. Wang, B.R.T. Simoneit, S. Shi, L. Zhang, F. Yang, Qualitative and quantitative analysis of dibenzothiophene, its methylated homologues, and benzonaphthothiophenes in crude oils, coal, and sediment extracts, J. Chromatogr. A 1233 (2012) 126-136, https://doi.org/10.1016/j.chroma.2012.01.086.

[28] R. Fang, M. Li, T.G. Wang, X. Liu, Y. Yuan, W. Jiang, D. Wang, S. Shi, Trimethyldibenzothiophenes: molecular tracers for filling pathways in oil reservoir, J. Petrol. Sci. Eng. 159 (2017) 451-460, https://doi.org/10.1016/j. petrol.2017.09.058.

[29] D. Carrizo, C. Domeno, I. Nerin, P. Alfaro, C. Nerin, Atmospheric pressure solid analysis probe coupled to quadrupole-time of flight mass spectrometry as a tool for screening and semi-quantitative approach of polycyclic aromatic hydrocarbons, nitro-polycyclic aromatic hydrocarbons and oxo-polycyclic aromatic, Talanta 131 (2015) 175-184, https://doi.org/10.1016/j.talanta.2014.07.034.

[30] P. Vera, E. Canellas, C. Nerin, Migration of odorous compounds from adhesives used in market samples of food packaging materials by chromatography olfactometry and mass spectrometry (GC-O-MS), Food Chem. 145 (2014) 237-244, https://doi.org/10.1016/j.foodchem.2013.06.087.
[31] C.P. Wu, K.N. Qian, C.C. Walters, A. Mennito, Application of atmospheric pressure ionization techniques and tandem mass spectrometry for the characterization of petroleum components, Int. J. Mass Spectrom. 377 (2015) 728-735, https://doi. org/10.1016/j.ijms.2014.08.019.

[32] R. Fang, T.G. Wang, M. Li, Z. Xiao, B. Zhang, S. Huang, S. Shi, D. Wang, W. Deng, Dibenzothiophenes and benzo[b]naphthothiophenes: molecular markers for tracing oil filling pathways in the carbonate reservoir of the Tarim Basin, NW China, Org. Geochem. 91 (2016) 68-80, https://doi.org/10.1016/j. orggeochem.2015.11.004.

[33] D. Stevens, Q. Shi, C.S. Hsu, Novel analytical technique for petroleum biomarker analysis, Energy Fuel. 27 (2013) 167-171, https://doi.org/10.1021/ef301645g.

[34] C. Domeno, E. Canellas, P. Alfaro, A. Rodriguez-Lafuente, C. Nerin, Atmospheric pressure gas chromatography with quadrupole time of flight mass spectrometry for simultaneous detection and quantification of polycyclic aromatic hydrocarbons and nitro-polycyclic aromatic hydrocarbons in mosses, J. Chromatogr. A 1252 (2012) 146-154, https://doi.org/10.1016/j.chroma.2012.06.061.

[35] N.E. Moustafa, J.T. Andersson, Analysis of polycyclic aromatic sulfur heterocycles in Egyptian petroleum condensate and volatile oils by gas chromatography with atomic emission detection, Fuel Process. Technol. 92 (2011) 547-555, https://doi. org/10.1016/j.fuproc.2010.11.010.

[36] C. Yang, Z. Yang, G. Zhang, B. Hollebone, M. Landriault, Z. Wang, P. Lambert, C. E. Brown, Characterization and differentiation of chemical fingerprints of virgin and used lubricating oils for identification of contamination or adulteration sources, Fuel 163 (2016) 271-281, https://doi.org/10.1016/j.fuel.2015.09.070.

[37] M. Asif, R. Alexander, T. Fazeelat, K. Pierce, Geosynthesis of dibenzothiophene and alkyl dibenzothiophenes in crude oils and sediments by carbon catalysis, Org. Geochem. 40 (2009) 895-901, https://doi.org/10.1016/j. orggeochem.2009.04.016.

[38] A.R. García-Cicourel, B. van de Velde, G. Roskam, H.G. Janssen, Supercritical fluid chromatography as a rapid single-step method for the determination of mineral oil saturated and aromatic hydrocarbons in purified mineral oils for food and cosmetics applications, J. Chromatogr. A 1614 (2020), https://doi.org/10.1016/j. chroma.2019.460713.

[39] Z. Wang, M.F. Fingas, Development of oil hydrocarbon fingerprinting and identification techniques, Mar. Pollut. Bull. 47 (2003) 423-452, https://doi.org/ 10.1016/S0025-326X(03)00215-7.

[40] I. Zrafi, L. Hizem, H. Chalghmi, A. Ghrabi, M. Rouabhia, D. Saidane-Mosbahi, Aliphatic and aromatic biomarkers for petroleum hydrocarbon investigation in marine sediment, Journal of Petroleum Science Research 2 (2013) 145, https:// doi.org/10.14355/jpsr.2013.0204.01. 